\title{
About viscous approximations of the bitemperature Euler system
}

\author{
D. Aregba-Driollet, S. Brull
}

\begin{abstract}
This paper is devoted to the study of the construction of a viscous approximation of the nonconservative bitemperature Euler system. Starting from a BGK model coupled with Ampère and Poisson equations proposed in [1], we perform a Chapman-Enskog expansion up to order 1 leading to a Navier-Stokes system. Next, we prove that this system is compatible with the entropy of the bitemperature Euler system.
\end{abstract}

\section{Introduction}

This paper is devoted to a viscous approximation of the bitemperature Euler system that has been studied in [1]. This fluid model describes the interaction of a mixture of one species of ions and one species of electrons in thermal nonequilibrium, with applications in the field of Inertial Confinement Fusion where solutions with shocks occur. Quasineutrality being assumed, the electronic and ionic mass fractions are constant: subscripts $e$ and $i$ standing for electron and ions respectively,

$$
\rho_{e}=m_{e} n_{e}=c_{e} \rho, \quad \rho_{i}=m_{i} n_{i}=c_{i} \rho, \quad c_{e}+c_{i}=1
$$

and the model consists of two conservation equations for mass and momentum and two nonconservative equations for each energy.

Moreover the pressure of each species is supposed to satisfy a gamma-law with its own $\gamma$ constant:

$$
p_{e}=\left(\gamma_{e}-1\right) \rho_{e} \varepsilon_{e}=n_{e} k_{B} T_{e}, \quad p_{i}=\left(\gamma_{i}-1\right) \rho_{i} \varepsilon_{i}=n_{i} k_{B} T_{i},
$$

where $k_{B}$ is the Boltzmann constant, $\varepsilon_{\alpha}$ and $T_{\alpha}$ represent respectively the internal specific energy and the temperature of species $\alpha, \alpha \in\{e, i\}$.

The total energies are given by $\mathcal{E}_{\alpha}=\rho_{\alpha} \varepsilon_{\alpha}+\frac{1}{2} \rho_{\alpha} u^{2}, \alpha \in\{e, i\}$. We denote $\nu_{e i} \geq 0$ the interaction coefficient between electronic and ionic temperatures. The bitemperature Euler system is the following:

$$
\left\{\begin{array}{l}
\partial_{t} \rho+\partial_{x}(\rho u)=0 \\
\partial_{t}(\rho u)+\partial_{x}\left(\rho u^{2}+p_{e}+p_{i}\right)=0 \\
\partial_{t} \mathcal{E}_{e}+\partial_{x}\left(u\left(\mathcal{E}_{e}+p_{e}\right)\right)-u\left(c_{i} \partial_{x} p_{e}-c_{e} \partial_{x} p_{i}\right)=\nu_{e i}\left(T_{i}-T_{e}\right) \\
\partial_{t} \mathcal{E}_{i}+\partial_{x}\left(u\left(\mathcal{E}_{i}+p_{i}\right)\right)+u\left(c_{i} \partial_{x} p_{e}-c_{e} \partial_{x} p_{i}\right)=-\nu_{e i}\left(T_{i}-T_{e}\right)
\end{array}\right.
$$


A first step in the comprehension of this system is to suppose that $\gamma_{e}=\gamma_{i}=\gamma$. In this case, one can define a global internal energy $\varepsilon=c_{e} \varepsilon_{e}+c_{i} \varepsilon_{i}$ which satisfies $p_{e}+p_{i}=(\gamma-1) \rho \varepsilon$. Denoting $\mathcal{E}=\mathcal{E}_{e}+\mathcal{E}_{i}$ the total energy one has

$$
\mathcal{E}=\rho \varepsilon+\frac{1}{2} \rho u^{2}
$$

and

$$
\partial_{t} \mathcal{E}+\partial_{x}(u(\mathcal{E}+p))=0
$$

so that $(\rho, \rho u, \mathcal{E})$ satisfies the usual Euler $3 \times 3$ system with $\gamma$ law. Nevertheless, even in this case one needs to solve a nonconservative equation in order to get $T_{e}$ and $T_{i}$ separately. In our context, the nonconservativity is not only due to source terms but especially to terms multiplying $u$ by pressure gradients, making delicate the definition of admissible shocks. In order to define nonconservative products, Dal Maso, Le Floch and Murat proposed in [9] a new theory based on the definition of family of paths. In [7], the authors consider the bitemperature Euler system with diffusive terms. By assuming that the electrons are isentropic, the system is transformed into a conservative model. In [13], the authors consider a kinetic system for sprays and derive a nonconservative hyperbolic system that is studied in [11].

In [1], the Euler bitemperature system has been derived by hydrodynamic limit of an underlying kinetic model which consists of a BGK model coupled with Poisson equation in the quasineutral regime. Moreover the obtained fluid system have been proved to be entropy dissipative by a direct approach and also by using the Boltzmann entropy. In particular, the nonconservative terms are obtained from the definition of the electric field according to a generalized Ohm's law. Numerical simulations have been performed with a finite volume scheme designed from this kinetic model, and have been proved to be physically realistic. Relaxation schemes based on the Suliciu approach have also been developped in [1] for this sytem and in [5] in the case of a transverse magnetic configuration. In [15], the authors perform a Chapman-Enskog expansion by introducing a small parameter representing the ratio between electronic and ionic molecular masses in the spirit of [10]. They obtain an hyperbolic system with a parabolic regularisation on the electrons.

One of the motivation of the derivation of viscous terms is the construction of traveling waves for the determination of shock profiles. In particular, in the context of a nonconservative system for two-phase flows, the authors construct in ([11], [12]) traveling waves solutions. The construction of traveling waves is also considered in [15] by using a diffusive equations on the electrons. Therefore this work is a first step before the construction of travelling waves for the bitemperature Euler system.

More precisely, in the present paper, we perform a Chapman-Enskog expansion of our kinetic model up to order one in order to get rigorously a viscous, Navier-Stokes type approximation of the bitemperature Euler system in the case $\gamma_{e}=\gamma_{i}$. As a result, we obtain conservative and nonconservative second order terms. To go into details, let us denote $\mathcal{U}=\left(\rho, \rho u, \mathcal{E}_{e}, \mathcal{E}_{i}\right)$. The Euler bitemperature system (1.2) being written in condensed form as

$$
\partial_{t} \mathcal{U}+A(\mathcal{U}) \partial_{x} \mathcal{U}=S(\mathcal{U}),
$$

for a fixed relaxation parameter $\tau>0$ the obtained second order system can be written under 
the form

$$
\partial_{t} \mathcal{U}^{\tau}+A\left(\mathcal{U}^{\tau}\right) \partial_{x} \mathcal{U}^{\tau}=S\left(\mathcal{U}^{\tau}\right)+\tau\left(u^{\tau} \partial_{x}\left(J\left(\mathcal{U}^{\tau}\right) \partial_{x} \mathcal{U}^{\tau}\right)+\partial_{x}\left(D\left(\mathcal{U}^{\tau}\right) \partial_{x} \mathcal{U}^{\tau}\right)\right)
$$

Here $J\left(\mathcal{U}^{\tau}\right)$ and $D\left(\mathcal{U}^{\tau}\right)$ are $4 \times 4$ matrices, while $u^{\tau}$ is the velocity. This result completes known models such as the one studied by $\mathrm{C}$. Chalons and F. Coquel in [6], by constructing rigorously some second order terms to their system. Next we prove the compatibility of the entropy of the bitemperature Euler system with the diffusive terms. We recall that a dissipative entropy $\eta$ exists for (1.2), namely

$$
\eta(\mathcal{U})=\bar{\eta}_{e}\left(\rho c_{e}, \varepsilon_{e}\right)+\bar{\eta}_{i}\left(\rho c_{i}, \varepsilon_{i}\right), \quad \bar{\eta}_{\alpha}\left(\rho_{\alpha}, \varepsilon_{\alpha}\right)=-\frac{\rho_{\alpha}}{m_{\alpha}\left(\gamma_{\alpha}-1\right)} \ln \left(\frac{p_{\alpha}}{\rho_{\alpha}^{\gamma_{\alpha}}}\right), \quad \alpha \in\{e, i\}
$$

with flux $Q(\mathcal{U})=u \eta(\mathcal{U})[1]$. We prove here that the solutions $\mathcal{U}^{\tau}$ of $(1.3)$ formally satisfy the following inequality:

$$
\partial_{t} \eta\left(\mathcal{U}^{\tau}\right)+\partial_{x}\left(u \eta\left(\mathcal{U}^{\tau}\right)\right) \leq-\frac{\nu_{e i}}{k_{B} T_{i} T_{e}}\left(T_{i}-T_{e}\right)^{2}-\tau \frac{5 k_{B}}{2 m_{\alpha}} \sum_{\alpha=e, i} \partial_{x}\left(n_{\alpha} \partial_{x} T_{\alpha}\right) .
$$

As a consequence, if $\left(\mathcal{U}^{\tau}\right)_{\tau}$ is smooth and decreases fast enough at infinity one has

$$
\frac{d}{d t} \int_{\mathbb{R}} \eta\left(\mathcal{U}^{\tau}(x, t)\right) d x \leq-\frac{\nu_{e i}}{k_{B}} \int_{\mathbb{R}} \frac{\left(T_{i}^{\tau}-T_{e}^{\tau}\right)^{2}}{T_{i}^{\tau} T_{e}^{\tau}} d x .
$$

This is a first step to prove that $\mathcal{U}^{\tau}$ owns a limit $\mathcal{U}$ which is a weak entropy solution of the Euler bitemperature system, see [14] for detailed argumentation. In the limit we have

$$
\partial_{t} \eta(\mathcal{U})+\partial_{x} Q(\mathcal{U}) \leq-\frac{\nu_{e i}}{k_{B} T_{i} T_{e}}\left(T_{i}-T_{e}\right)^{2} .
$$

This inequality is the one satisfied formally by a limit of the moments of the solution of the BGK system [1].

In the case of BGK approximations of systems of conservation laws, the entropy dissipativity of the Chapman-Enskog expansion is wellknown, see [4], [8]. As a matter of fact if $\mathcal{F}$ is the flux function of such a system, the Chapman-Enskog expansion is

$$
\partial_{t} U^{\tau}+\partial_{x} \mathcal{F}\left(U^{\tau}\right)=\tau \partial_{x}\left(D\left(U^{\tau}\right) \partial_{x} U^{\tau}\right)
$$

with

$$
D(U)=\Psi^{\prime}(U)-\mathcal{F}^{\prime}(u) \circ \mathcal{F}^{\prime}(u),
$$

the matrix $\Psi^{\prime}(U)$ depending on the chosen BGK framework. Then one multiplies (1.6) by $\eta^{\prime}\left(U^{\tau}\right)$ and uses the equality

$$
\eta^{\prime}(U) \partial_{x}\left(D(U) \partial_{x} U\right)=\partial_{x}\left(\eta^{\prime}(U) D(U) \partial_{x} U\right)-\eta^{\prime \prime}(U)\left(\partial_{x} U, D(U) \partial_{x} U\right) .
$$

In this case, ${ }^{t} D(U) \eta^{\prime \prime}(U)$ is symmetric nonnegative so that

$$
\partial_{t} \eta\left(\mathcal{U}^{\tau}\right)+\partial_{x} Q\left(\mathcal{U}^{\tau}\right) \leq \tau \partial_{x}\left(\eta^{\prime}\left(U^{\tau}\right) D\left(U^{\tau}\right) \partial_{x} U^{\tau}\right) .
$$


In the case of the present paper, $A(\mathcal{U})$ is not a gradient, so we had to perform a direct calculation to prove the dissipation property.

The paper is organized as follows. The Section 2 deals with the derivation of a Navier-Stokes system starting from the kinetic system proposed in [1]. In section 3 , the diffusive terms are shown to be dissipative w.r.t. the entropy of the bitemperature Euler system. Finally, section 4 gives conclusions to this work.

\section{Derivation of the Navier-Stokes system}

\subsection{Notations}

Kinetic models are described by the distribution function $f_{\alpha}$ of each species depending on the time variable $t \in \mathbb{R}_{+}$, on the position $x \in \mathbb{R}^{3}$ and on the velocity $v \in \mathbb{R}^{3}$. The macroscopic quantities can be obtained by extracting moments on these distribution functions w.r.t the velocity variable. Indeed density, velocity and total energy of the species $\alpha$ can be defined as

$$
n_{\alpha}=\int_{\mathbb{R}^{3}} f_{\alpha} d v, \quad u_{\alpha}=\frac{1}{n_{\alpha}} \int_{\mathbb{R}^{3}} v_{1} f_{\alpha} d v, \quad \mathcal{E}_{\alpha}=\frac{3}{2} \rho_{\alpha} \frac{k_{B}}{m_{\alpha}} T_{\alpha}+\frac{1}{2} \rho_{\alpha} u_{\alpha}^{2}=\int_{\mathbb{R}^{3}} m_{\alpha} \frac{v^{2}}{2} f_{\alpha} d v .
$$

The present model is monoatomic $\left(\gamma=\frac{5}{3}\right)$. Hence, the internal specific energy of species $\alpha$ writes

$$
\varepsilon_{\alpha}=\frac{3}{2 m_{\alpha}} k_{B} T_{\alpha}
$$

In the following we shall use the moment operator $P_{\alpha}$ defined by

$$
P_{\alpha}\left(f_{\alpha}\right)=m_{\alpha} \int_{\mathbb{R}^{3}}\left(\begin{array}{c}
1 \\
v_{1} \\
\frac{v^{2}}{2}
\end{array}\right) f_{\alpha} d v .
$$

We denote $P_{\alpha}\left(f_{\alpha}\right)=U_{\alpha}$ :

$$
U_{\alpha}=\left(\begin{array}{c}
\rho_{\alpha} \\
\rho_{\alpha} u_{\alpha} \\
\mathcal{E}_{\alpha}
\end{array}\right) .
$$

Usually the velocity and the temperature of the mixture are defined by

$$
u=\frac{\rho_{e} u_{e}+\rho_{i} u_{i}}{\rho_{e}+\rho_{i}}, \quad n k_{B} T=\sum_{\alpha}\left(\frac{1}{2} \rho_{\alpha}\left(u_{\alpha}^{2}-u^{2}\right)\right)+\sum_{\alpha}\left(n_{\alpha} k_{B} T_{\alpha}\right),
$$

where $n=n_{e}+n_{i}$.

Moreover, the current of the plasma $j$ and the total charge $\bar{\rho}$ are defined by

$$
\bar{\rho}=\int_{\mathbb{R}^{3}}\left(q_{e} f_{e}+q_{i} f_{i}\right) d v=n_{e} q_{e}+n_{i} q_{i}, \quad j=\int_{\mathbb{R}^{3}} v_{1}\left(q_{e} f_{e}+q_{i} f_{i}\right) d v=n_{e} q_{e} u_{e}+n_{i} q_{i} u_{i} .
$$




\subsection{Chapman-Enskog expansion}

Consider the following kinetic model in the quasi-neutral regime

$$
\left\{\begin{array}{cl}
\partial_{t} f_{\alpha}+v_{1} \partial_{x} f_{\alpha}+\frac{q_{\alpha}}{m_{\alpha}} E \partial_{v_{1}} f_{\alpha} & =\frac{1}{\tau}\left(\mathcal{M}_{\alpha}-f_{\alpha}\right)+\frac{1}{\tau_{e i}}\left(\overline{\mathcal{M}_{\alpha}}-f_{\alpha}\right), \\
\partial_{t} E & =-\frac{j}{\tau^{2}}, \\
\partial_{x} E & =\frac{\rho}{\tau^{2}},
\end{array}\right.
$$

where $\tau$ is a positive parameter proportional to the Knudsen number.

$\mathcal{M}_{\alpha}$ and $\overline{\mathcal{M}_{\alpha}}$ are the two Maxwellian distribution functions

$$
\begin{aligned}
& \mathcal{M}_{\alpha}\left(f_{\alpha}\right)=\frac{n_{\alpha}}{\left(2 \pi k_{B} T_{\alpha} / m_{\alpha}\right)^{3 / 2}} \exp \left(-\frac{\left|v-u_{\alpha}\right|^{2}}{2 k_{B} T_{\alpha} / m_{\alpha}}\right), \alpha=e, i, \\
& \overline{\mathcal{M}_{\alpha}}\left(f_{e}, f_{i}\right)=\frac{n_{\alpha}}{\left(2 \pi k_{B} T / m_{\alpha}\right)^{3 / 2}} \exp \left(-\frac{|v-u|^{2}}{2 k_{B} T / m_{\alpha}}\right), \alpha=e, i .
\end{aligned}
$$

Next we perform a first order Chapman-Engskog expansion up to order 1. Hence the solution of the system $(2.12,2.13,2.14) f_{\alpha}$ is researched as the expansion

$$
f_{\alpha}=\mathcal{M}_{\alpha}+\tau g_{\alpha}, \quad \alpha \in\{e, i\}
$$

with the constraints

$$
\int_{\mathbb{R}^{3}} f_{\alpha} d v=\int_{\mathbb{R}^{3}} \mathcal{M}_{\alpha} d v, \int_{\mathbb{R}^{3}} v_{1} f_{\alpha} d v=\int_{\mathbb{R}^{3}} v_{1} \mathcal{M}_{\alpha} d v, \int_{\mathbb{R}^{3}} v^{2} f_{\alpha} d v=\int_{\mathbb{R}^{3}} v^{2} \mathcal{M}_{\alpha} d v
$$

So

$$
\int_{\mathbb{R}^{3}} g_{\alpha} d v=0, \int_{\mathbb{R}^{3}} v_{1} g_{\alpha} d v=0, \int_{\mathbb{R}^{3}} v^{2} g_{\alpha} d v=0
$$

\subsection{Euler system}

At this level, it is possible to compute the Euler system by arguing as in ([1]). Hence by plugging the expansion $(2.15,2.16,2.17)$ into $(2.12)$ and considering terms up to order 1 , it holds that

$$
\partial_{t} \mathcal{M}_{\alpha}+v_{1} \partial_{x} \mathcal{M}_{\alpha}+\frac{q_{\alpha}}{m_{\alpha}} E \partial_{v_{1}} \mathcal{M}_{\alpha}=-g_{\alpha}+\frac{1}{\tau_{\alpha \beta}}\left(\overline{\mathcal{M}}_{\alpha}-\mathcal{M}_{\alpha}\right)+\mathcal{O}(\tau)
$$

with $j=\mathcal{O}\left(\tau^{2}\right)$ and $\bar{\rho}=\mathcal{O}\left(\tau^{2}\right)$. Hence by using that

$$
\int_{\mathbb{R}^{3}} m_{\alpha} \mathcal{M}_{\alpha} d v=\rho_{\alpha}, \quad \int_{\mathbb{R}^{3}} m_{\alpha} v_{1} \mathcal{M}_{\alpha} d v=\rho_{\alpha} u, \quad \int_{\mathbb{R}^{3}} m_{\alpha} v^{2} \mathcal{M}_{\alpha} d v=\rho_{\alpha} \varepsilon_{\alpha}+\frac{1}{2} \rho_{\alpha} u^{2},
$$

we get

$$
\left\{\begin{array}{ccc}
\partial_{t} \rho+\partial_{x}(\rho u) & = & \mathcal{O}(\tau), \\
\partial_{t}(\rho u)+\partial_{x}\left(\rho u^{2}+p_{e}+p_{i}\right) & = & \mathcal{O}(\tau), \\
\partial_{t}\left(\rho_{e} \varepsilon_{e}+\frac{1}{2} \rho_{e} u^{2}\right)+\partial_{x}\left(u\left(\rho_{e} \varepsilon_{e}+\frac{1}{2} \rho_{e} u^{2}+p_{e}\right)\right)-u\left(c_{i} \partial_{x} p_{e}-c_{e} \partial_{x} p_{i}\right) & = & \nu_{e i}\left(T_{i}-T_{e}\right)+\mathcal{O}(\tau), \\
\partial_{t}\left(\rho_{i} \varepsilon_{i}+\frac{1}{2} \rho_{i} u^{2}\right)+\partial_{x}\left(u\left(\rho_{i} \varepsilon_{i}+\frac{1}{2} \rho_{i} u^{2}+p_{i}\right)\right)+u\left(c_{i} \partial_{x} p_{e}-c_{e} \partial_{x} p_{i}\right) & = & -\nu_{e i}\left(T_{i}-T_{e}\right)+\mathcal{O}(\tau) .
\end{array}\right.
$$




\subsection{Obtention of the viscous fluid system}

We expand $f_{\alpha}$ as in $(2.15,2.16,2.17)$ and we extract the moments $w . r . t .1, v, v^{2}$.

One important point to determine the viscous terms of the Navier-Stokes is to compute the term $g_{\alpha}$ of the expansion $\left.(2.15,2.16,2.17)\right)$. The calculus is performed in the following proposition.

Proposition 1. The first order terms $g_{e}$ and $g_{i}$ of the expansion (2.15, 2.16, 2.17)) write

$$
\begin{aligned}
g_{e} & =-\left(\left(\left(v_{1}-u\right)\left(\frac{\partial_{x} n_{e}}{n_{e}}-\frac{3}{2} \frac{\partial_{x} T_{e}}{T_{e}}\right)+\partial_{x} u\left(\frac{\left(v_{1}-u\right)^{2}}{\frac{k_{B}}{m_{e}} T_{e}}-\frac{1}{3} \frac{(v-u)^{2}}{\frac{k_{B}}{m_{e}} T_{e}}\right)\right.\right. \\
& \left.+\frac{\nu_{e i}}{n_{e} k_{B} T_{e}}\left(T_{i}-T_{e}\right)\left(\frac{(v-u)^{2}}{3 \frac{k_{B}}{m_{e}} T_{e}}-1\right)-\left(v_{1}-u\right) \frac{(v-u)^{2}}{2 \frac{k_{B}}{m_{e}}} \partial_{x}\left(\frac{1}{T_{e}}\right)-\frac{\left(v_{1}-u\right)}{\frac{k_{B}}{m_{e}} T_{e} \rho} \partial_{x}\left(p_{e}+p_{i}\right)\right) \mathcal{M}_{e} \\
& \left.+\frac{q_{e}}{m_{e}} E \partial_{v_{1}} \mathcal{M}_{e}-\frac{1}{\tau_{e i}}\left(\overline{\mathcal{M}}_{e}-\mathcal{M}_{e}\right)\right) \\
g_{i} & =-\left(\left(\left(v_{1}-u\right)\left(\frac{\partial_{x} n_{i}}{n_{i}}-\frac{3}{2} \frac{\partial_{x} T_{i}}{T_{i}}\right)+\partial_{x} u\left(\frac{\left(v_{1}-u\right)^{2}}{\frac{k_{B}}{m_{i}} T_{i}}-\frac{1}{3} \frac{(v-u)^{2}}{\frac{k_{B}}{m_{i}} T_{i}}\right)\right.\right. \\
& \left.+\frac{\nu_{e i}}{n_{i} k_{B} T_{i}}\left(T_{e}-T_{i}\right)\left(\frac{(v-u)^{2}}{3 \frac{k_{B}}{m_{i}} T_{i}}-1\right)-\left(v_{1}-u\right) \frac{(v-u)^{2}}{2 \frac{k_{B}}{m_{i}}} \partial_{x}\left(\frac{1}{T_{i}}\right)-\frac{\left(v_{1}-u\right)}{\frac{k_{B}}{m_{i}} T_{i} \rho} \partial_{x}\left(p_{e}+p_{i}\right)\right) \mathcal{M}_{i} \\
& \left.+\frac{q_{i}}{m_{i}} E \partial_{v_{1}} \mathcal{M}_{i}-\frac{1}{\tau_{e i}}\left(\overline{\mathcal{M}}_{i}-\mathcal{M}_{i}\right)\right) .
\end{aligned}
$$

Proof. $g_{\alpha}$ is given by the relation

$$
g_{\alpha}=-\left(\partial_{t} \mathcal{M}_{\alpha}+v_{1} \partial_{x} \mathcal{M}_{\alpha}+\frac{q_{\alpha}}{m_{\alpha}} E \partial_{v_{1}} \mathcal{M}_{\alpha}-\frac{1}{\tau_{e i}}\left(\overline{\mathcal{M}_{\alpha}}-\mathcal{M}_{\alpha}\right)\right.
$$

A direct computation gives

$$
\partial_{t} \mathcal{M}_{e}=\left(\left(\frac{\partial_{t} n_{e}}{n_{e}}-\frac{3}{2} \frac{\partial_{t} T_{e}}{T_{e}}\right)+\left(v_{1}-u\right) \frac{\partial_{t} u}{\frac{k_{B}}{m_{e}} T_{e}}-\frac{(v-u)^{2}}{2 \frac{k_{B}}{m_{e}} T_{e}} \partial_{t}\left(\frac{1}{T_{e}}\right)\right) \mathcal{M}_{e}
$$

and

$$
v_{1} \partial_{x} \mathcal{M}_{e}=\left(\left(\frac{v_{1} \partial_{x} n_{e}}{n_{e}}-\frac{3}{2} \frac{v_{1} \partial_{x} T_{e}}{T_{e}}\right)+\left(v_{1}-u\right) \frac{v_{1} \partial_{x} u}{\frac{k_{B}}{m_{e}} T_{e}}-\frac{(v-u)^{2}}{2 \frac{k_{B}}{m_{e}} T_{e}} v_{1} \partial_{x}\left(\frac{1}{T_{e}}\right)\right) \mathcal{M}_{e}
$$

By using the non-conservative Euler system (2.18), the time derivatives of (2.21) are computed 
in function of the space derivatives up to $\mathcal{O}(\tau)$ terms, as follows

$$
\begin{aligned}
\frac{\partial_{t} n_{e}}{n_{e}}-\frac{3}{2} \frac{\partial_{t} T_{e}}{T_{e}} & =-u \frac{\partial_{x} n_{e}}{n_{e}}+\frac{3}{2} u \frac{\partial_{x} T_{e}}{T_{e}}-\frac{\nu_{e i}}{n_{e} k_{B} T_{e}}\left(T_{i}-T_{e}\right)+\mathcal{O}(\tau), \\
\partial_{t} u & =-u \partial_{x} u-\frac{1}{\rho} \partial_{x}\left(p_{e}+p_{i}\right)+\mathcal{O}(\tau), \\
\frac{\partial_{t} n_{i}}{n_{i}}-\frac{3}{2} \frac{\partial_{t} T_{i}}{T_{i}} & =-u \frac{\partial_{x} n_{i}}{n_{i}}+\frac{3}{2} u \frac{\partial_{x} T_{i}}{T_{i}}-\frac{\nu_{e i}}{n_{i} k_{B} T_{i}}\left(T_{e}-T_{i}\right)+\mathcal{O}(\tau), \\
\frac{\partial_{t} T_{e}}{T_{e}} & =-u \frac{\partial_{x} T_{e}}{T_{e}}-\frac{2}{3} \partial_{x} u+\frac{2}{3} \frac{\nu_{e i}}{n_{e} k_{B} T_{e}}\left(T_{i}-T_{e}\right)+\mathcal{O}(\tau) \\
\frac{\partial_{t} T_{i}}{T_{i}} & =-u \frac{\partial_{x} T_{i}}{T_{i}}-\frac{2}{3} \partial_{x} u+\frac{2}{3} \frac{\nu_{e i}}{n_{i} k_{B} T_{i}}\left(T_{e}-T_{i}\right)+\mathcal{O}(\tau) .
\end{aligned}
$$

Hence up to $\mathcal{O}(\tau)$ order terms, we get

$$
\begin{aligned}
\partial_{t} \mathcal{M}_{e}+v_{1} \partial_{x} \mathcal{M}_{e} & =\left(-u \frac{\partial_{x} n_{e}}{n_{e}}+\frac{3}{2} u \frac{\partial_{x} T_{e}}{T_{e}}-\frac{\nu_{e i}}{n_{e} k_{B} T_{e}}\left(T_{i}-T_{e}\right)+\frac{\left(v_{1}-u\right)}{\frac{k_{B}}{m_{e}} T_{e}}\left(-u \partial_{x} u-\frac{1}{\rho} \partial_{x}\left(p_{e}+p_{i}\right)\right)\right. \\
& \left.+\frac{(v-u)^{2}}{2 \frac{k_{B}}{m_{e}} T_{e}}\left(-u \frac{\partial_{x} T_{e}}{T_{e}}-\frac{2}{3} \partial_{x} u+\frac{2}{3} \frac{\nu_{e i}}{n_{e} k_{B} T_{e}}\left(T_{i}-T_{e}\right)\right)\right) \mathcal{M}_{e}
\end{aligned}
$$

Therefore, we obtain

$$
\begin{aligned}
\partial_{t} \mathcal{M}_{e}+v_{1} \partial_{x} \mathcal{M}_{e} & =\left(\left(v_{1}-u\right)\left(\frac{\partial_{x} n_{e}}{n_{e}}-\frac{3}{2} \frac{\partial_{x} T_{e}}{T_{e}}\right)+\partial_{x} u\left(\frac{\left(v_{1}-u\right)^{2}}{\frac{k_{B}}{m_{e}} T_{e}}-\frac{(v-u)^{2}}{3 \frac{k_{B}}{m_{e}} T_{e}}\right)\right. \\
& +\frac{\nu_{e i}}{n_{e} k_{B} T_{e}}\left(T_{i}-T_{e}\right)\left(\frac{(v-u)^{2}}{3 \frac{k_{B}}{m_{e}} T_{e}}-1\right)-\left(v_{1}-u\right) \frac{(v-u)^{2}}{2 \frac{k_{B}}{m_{e}}} \partial_{x}\left(\frac{1}{T_{e}}\right) \\
& \left.-\frac{\left(v_{1}-u\right)}{\frac{k_{B}}{m_{e}} \rho T_{e}} \partial_{x}\left(p_{e}+p_{i}\right)\right) \mathcal{M}_{e}
\end{aligned}
$$

and we recover (2.19). The same result holds for (2.20). 
Proposition 2. The viscous approximation of the kinetic system (2.12, 2.13, 2.14) writes

$$
\begin{array}{r}
\partial_{t} \rho+\partial_{x}(\rho u)=0 \\
\partial_{t}(\rho u)+\partial_{x}\left(\rho u^{2}+p_{e}+p_{i}\right)-\frac{4}{3} \tau \partial_{x}\left(p \partial_{x} u\right)=0 \\
\partial_{t}\left(\rho_{e} \varepsilon_{e}+\frac{1}{2} \rho_{e} u^{2}\right)+\partial_{x}\left(u\left(\rho_{e} \varepsilon_{e}+\frac{1}{2} \rho_{e} u^{2}+p_{e}\right)\right)-u\left(c_{i} \partial_{x} p_{e}-c_{e} \partial_{x} p_{i}\right) \\
-u\left(\frac{4}{3} \tau c_{e} \partial_{x}\left(p_{i} \partial_{x} u\right)-\frac{4}{3} \tau c_{i} \partial_{x}\left(p_{e} \partial_{x} u\right)\right) \\
-\frac{4}{3} \tau \partial_{x}\left(u p_{e} \partial_{x} u\right)-\frac{5}{2} \tau \partial_{x}\left(\frac{k_{B}}{m_{e}} p_{e} \partial_{x} T_{e}\right)=\nu_{e i}\left(T_{e}-T_{i}\right) \\
\partial_{t}\left(\rho_{i} \varepsilon_{i}+\frac{1}{2} \rho_{i} u^{2}\right)+\partial_{x}\left(u\left(\rho_{i} \varepsilon_{i}+\frac{1}{2} \rho_{i} u^{2}+p_{i}\right)\right)+u\left(c_{i} \partial_{x} p_{e}-c_{e} \partial_{x} p_{i}\right) \\
+u\left(\frac{4}{3} \tau c_{e} \partial_{x}\left(p_{i} \partial_{x} u\right)-\frac{4}{3} \tau c_{i} \partial_{x}\left(p_{e} \partial_{x} u\right)\right) \\
-\frac{4}{3} \tau \partial_{x}\left(u p_{i} \partial_{x} u\right)-\frac{5}{2} \tau \partial_{x}\left(\frac{k_{B}}{m_{i}} p_{i} \partial_{x} T_{i}\right)=\nu_{e i}\left(T_{i}-T_{e}\right),
\end{array}
$$

where the electric field $E$ is given by

$$
\left(\frac{n_{e} q_{e}}{\rho_{e}}-\frac{n_{i} q_{i}}{\rho_{i}}\right) E=\frac{\rho}{\rho_{e} \rho_{i}} n_{e} q_{e} E=-\frac{\rho}{\rho_{e} \rho_{i}} n_{i} q_{i} E=\frac{\partial_{x} p_{e}}{\rho_{e}}-\frac{\partial_{x} p_{i}}{\rho_{i}}-\frac{4}{3} \frac{\tau}{\rho_{e}} \partial_{x}\left(p_{e} \partial_{x} u\right)+\frac{4}{3} \frac{\tau}{\rho_{i}} \partial_{x}\left(p_{i} \partial_{x} u\right) .
$$

Remark 1. In Proposition 2, the kinetic model is considered in the monoatomic case. This means that $\gamma_{e}=\gamma_{i}=\frac{5}{3}$. The polyatomic case can be managed by considering a kinetic model with internal energy variable like in [2].

Remark 2. The relation (2.26) is an approximation at order $\tau$ of the Ohm law given in [1]. The nonconservative terms of the system (2.22, 2.23, 2.24, 2.25) are shown to appear from this relation defining $E$

Proof. By expanding $f_{\alpha}$ like in (2.15), we get

$$
\partial_{t}\left(\mathcal{M}_{\alpha}+\tau g_{\alpha}\right)+v_{1} \partial_{x}\left(\mathcal{M}_{\alpha}+\tau g_{\alpha}\right)+\frac{q_{\alpha}}{m_{\alpha}} E \partial_{v_{1}}\left(\mathcal{M}_{\alpha}+\tau g_{\alpha}\right)=-g_{\alpha}+\frac{1}{\tau_{e i}}\left(\overline{\mathcal{M}}_{\alpha}-\mathcal{M}_{\alpha}-\tau g_{\alpha}\right)
$$

and we extract the moments of (2.27) w.r.t $1, v, v^{2}$. From the orthogonality conditions (2.17) the viscous part of the expansion will be given by the moments of $v_{1} \partial_{x} g_{\alpha}$ w.r.t $1, v, v^{2}$.

Firstly, we obtain the same mass conservation equation as for the Euler system because from the relation (2.17).

In order to get the impulsion equation (2.23), we compute

$$
\int_{\mathbb{R}^{3}} m_{e} v_{1}^{2} g_{e} d v=\int_{\mathbb{R}^{3}} m_{e}\left(v_{1}-u\right)^{2} g_{e} d v
$$


By using the expression of $g_{e}(2.19)$ and some orthogonality relations, it holds that

$$
\int_{\mathbb{R}^{3}} m_{e}\left(v_{1}-u\right)^{2} g_{e} d v=-\partial_{x} u \int_{\mathbb{R}^{3}}\left(\frac{\left(v_{1}-u\right)^{4}}{\frac{k_{B}}{m_{e}} T_{e}}-\frac{1}{3} \frac{\left(v_{1}-u\right)^{2}(v-u)^{2}}{\frac{k_{B}}{m_{e}} T_{e}}\right) \mathcal{M}_{e} m_{e} d v .
$$

Moreover, by using

$$
\int_{\mathbb{R}^{3}}(v-u)^{4} \mathcal{M}_{e} d v=n_{e}\left(\frac{k_{B} T_{e}}{m_{e}}\right)^{2},
$$

we get finally

$$
\int_{\mathbb{R}^{3}} m_{e}\left(v_{1}-u\right)^{2} g_{e} d v=-\frac{4}{3} p_{e} \partial_{x} u
$$

Hence we get for species $\alpha$

$$
\partial_{t}\left(\rho_{\alpha} u\right)+\partial_{x}\left(\rho_{\alpha} u+p_{\alpha}\right)-q_{\alpha} n_{\alpha} E-\frac{4}{3} \tau \partial_{x}\left(p_{\alpha} \partial_{x} u\right)=0 .
$$

Then by adding the previous equations for each species (2.23). Next in order to establish the relation defining $E,(2.29)$ is rewritten in the nonconservative form as

$$
\partial_{t} u+u \partial_{x} u+\frac{1}{\rho_{\alpha}} \partial_{x} p_{\alpha}-\frac{q_{\alpha} n_{\alpha}}{\rho_{\alpha}} E-\frac{4}{3} \frac{\tau}{\rho_{\alpha}} \partial_{x}\left(p_{\alpha} \partial_{x} u\right)=0 .
$$

By substracting equation (2.30) for electrons and ions and by proceeding like in [1], we get the generalized Ohm's law (2.26).

By using the relation (2.17), we obtain

$$
\int_{\mathbb{R}^{3}} \frac{m_{e}}{2} v_{1} v^{2} g_{e} d v=\int_{\mathbb{R}^{3}} \frac{m_{e}}{2}\left(v_{1}-u\right)(v-u)^{2} g_{e} d v+u \int_{\mathbb{R}^{3}} m_{e} v_{1}^{2} g_{e} d v .
$$

By using orthogonality relations, it comes that

$$
\begin{aligned}
\int_{\mathbb{R}^{3}} \frac{m_{e}}{2}\left(v_{1}-u\right)(v-u)^{2} g_{e} d v & =-5 \rho_{e}\left(\frac{k_{B}}{m_{e}} T_{e}\right)^{2}\left(\frac{\partial_{x} n_{e}}{n_{e}}-\frac{3}{2} \frac{\partial_{x} T_{e}}{T_{e}}\right)+\partial_{x}\left(\frac{1}{T_{e}}\right) \frac{35 \rho_{e}}{2 \frac{k_{B}}{m_{e}}}\left(\frac{k_{B}}{m_{e}} T_{e}\right)^{3} \\
& +\int_{\mathbb{R}^{3}}\left(v_{1}-u\right)^{2} \frac{(v-u)^{2}}{\frac{k_{B}}{m_{e}} \rho T_{e}} \partial_{x}\left(p_{e}+p_{i}\right) \mathcal{M}_{e} d v+5 n_{e} \frac{k_{B}}{m_{e}} T_{e} \frac{q_{e}}{m_{e}} E .
\end{aligned}
$$

Hence

$$
\begin{aligned}
\int_{\mathbb{R}^{3}} \frac{m_{e}}{2}\left(v_{1}-u\right)(v-u)^{2} g_{e} d v & =-5 \rho_{e}\left(\frac{k_{B}}{m_{e}} T_{e}\right)^{2}\left(\frac{\partial_{x} n_{e}}{n_{e}}-\frac{3}{2} \frac{\partial_{x} T_{e}}{T_{e}}\right)-\partial_{x} T_{e} \frac{35 \rho_{e}}{2 \frac{k_{B}}{m_{e}}}\left(\frac{k_{B}}{m_{e}}\right)^{3} T_{e} \\
& +5 \frac{\rho_{e}}{\frac{k_{B}}{m_{e}} \rho T_{e}}\left(\frac{k_{B}}{m_{e}} T_{e}\right)^{2} \partial_{x}\left(p_{e}+p_{i}\right)+5 n_{e} \frac{k_{B}}{m_{e}} T_{e} q_{e} E .
\end{aligned}
$$


Next, we use the generalized Ohm's law (2.26) that has been established previously. So neglecting the first order terms in $\tau$ in the expression of $E$, it comes that

$$
\begin{aligned}
\int_{\mathbb{R}^{3}} \frac{m_{e}}{2}\left(v_{1}-u\right)(v-u)^{2} g_{e} d v & =-5\left(\frac{k_{B}}{m_{e}} T_{e}\right)^{2} \partial_{x} n_{e}+\frac{15}{2} n_{e} \frac{k_{B}}{m_{e}} k_{B} \partial_{x} T_{e}-\frac{35}{2} k_{B} \frac{k_{B}}{m_{e}} T_{e} \partial_{x} T_{e} \\
& +5\left(\frac{k_{B}}{m_{e}} T_{e}\right) c_{e} \partial_{x}\left(p_{e}+p_{i}\right)+5 T_{e} \frac{k_{B}}{m_{e}}\left(c_{i} \partial_{x} p_{e}-c_{e} \partial_{x} p_{i}\right) .
\end{aligned}
$$

So

$$
\int_{\mathbb{R}^{3}} \frac{m_{e}}{2}\left(v_{1}-u\right)(v-u)^{2} g_{e} d v=-\frac{5}{2} \frac{k_{B}}{m_{e}} p_{e} \partial_{x} T_{e}
$$

and finally by using the relation (2.28), it holds that

$$
\int_{\mathbb{R}^{3}} \frac{m_{e}}{2} v_{1} v^{2} g_{e} d v=-\frac{4}{3} u p_{e} \partial_{x} u-\frac{5}{2} \frac{k_{B}}{m_{e}} p_{e} \partial_{x} T_{e} .
$$

So we obtain (2.24). The same result holds for (2.25)

\subsection{Navier-Stokes viscous system written with internal energy}

Next, in order to compare with ([3], [6]), the system $(2.22,2.23,2.24,2.25)$ is rewritten by using internal energies.

Proposition 3. The system (2.22, 2.23, 2.24, 2.25) is equivalent to the system

$$
\begin{array}{r}
\partial_{t} \rho+\partial_{x}(\rho u)=0, \\
\partial_{t}(\rho u)+\partial_{x}\left(\rho u^{2}+p_{e}+p_{i}\right)-\frac{4}{3} \tau \partial_{x}\left(p \partial_{x} u\right)=0, \\
\partial_{t}\left(\rho_{e} \varepsilon_{e}\right)+\partial_{x}\left(u\left(\rho_{e} \varepsilon_{e}\right)\right)+p_{e} \partial_{x} u+\frac{4}{3} \tau \partial_{x}\left(u p_{e} \partial_{x} u\right)-\frac{5}{2} \tau \partial_{x}\left(\frac{k_{B}}{m_{e}} p_{e} \partial_{x} T_{e}\right) \\
-\frac{4}{3} \tau p_{e}\left(\partial_{x} u\right)^{2}=\nu_{e i}\left(T_{e}-T_{i}\right), \\
\partial_{t}\left(\rho_{i} \varepsilon_{i}\right)+\partial_{x}\left(u\left(\rho_{i} \varepsilon_{i}\right)\right)+p_{i} \partial_{x} u+\frac{4}{3} \tau \partial_{x}\left(u p_{i} \partial_{x} u\right)-\frac{5}{2} \tau \partial_{x}\left(\frac{k_{B}}{m_{i}} p_{i} \partial_{x} T_{i}\right) \\
-\frac{4}{3} \tau p_{i}\left(\partial_{x} u\right)^{2}=\nu_{e i}\left(T_{i}-T_{e}\right) .
\end{array}
$$

Proof. Firstly, a straightforward computation gives

$$
\begin{aligned}
\frac{1}{2} \partial_{t}\left(\rho_{i} u^{2}\right) & =-\frac{1}{2} \partial_{x}\left(\rho u^{3}\right)-u \partial_{x} p_{i}+q_{i} n_{i} u E+\frac{4}{3} \tau u \partial_{x}\left(p_{i} \partial_{x} u\right) \\
& =-\partial_{x}\left(u\left(\frac{1}{2} \rho_{i} u^{2}+p_{i}\right)+p_{i} \partial_{x} u+q_{i} n_{i} u E+\frac{4}{3} \tau u \partial_{x}\left(p_{i} \partial_{x} u\right)\right.
\end{aligned}
$$

Hence, by using the viscous Ohm's law (2.26), we get the relation

$$
u n_{i} q_{i} E=-u c_{i} \partial_{x} p_{e}+u c_{e} \partial_{x} p_{i}+\frac{4}{3} c_{i} \tau u \partial_{x}\left(p_{e} \partial_{x} u\right)-\frac{4}{3} c_{e} \tau u \partial_{x}\left(p_{i} \partial_{x} u\right) .
$$


Therefore

$$
\begin{aligned}
\partial_{t}\left(\rho_{i} \varepsilon_{i}+\frac{1}{2} \rho_{i} u^{2}\right)+\partial_{x}\left(u\left(\rho_{i} \varepsilon_{i}+\frac{1}{2} \rho_{i} u^{2}+p_{i}\right)\right. & =\partial_{t}\left(\rho_{i} \varepsilon_{i}\right)+\partial_{x}\left(u \rho_{i} \varepsilon_{i}\right)+p_{i} \partial_{x} u \\
& -u c_{i} \partial_{x} p_{e}+u c_{e} \partial_{x} p_{i}+\frac{4}{3} c_{i} u \tau \partial_{x}\left(p \partial_{x} u\right) .
\end{aligned}
$$

Hence from the relation

$$
\partial_{x}\left(u p_{i} \partial_{x} u\right)=u \partial_{x}\left(p_{i} \partial_{x} u\right)+p_{i}\left(\partial_{x} u\right)^{2},
$$

we obtain the equation (2.34).

We firstly remark that the viscous system of ([3], [6]) contains the terms $p_{\alpha} \partial_{x} u$ and $-\frac{4}{3} \tau p_{\alpha}\left(\partial_{x} u\right)^{2}$, for $\alpha \in\{e ; i\}$. However, the terms $\tau \frac{4}{3} \partial_{x}\left(u p_{\alpha} \partial_{x} u\right)$ and $-\frac{5}{2} \tau \partial_{x}\left(\frac{k_{B}}{m_{i}} p_{i} \partial_{x} T_{i}\right)$ do not appear in ([3], $[6])$.

\section{Dissipativity of the second order terms with respect to the entropy}

This section is devoted to the proof of the entropy dissipativity of the viscous system (2.22-2.25).

Proposition 4. We assume that $\gamma_{e}=\gamma_{i}=5 / 3$. Let $\mathcal{U}^{\tau}$ be a solution of the second order system (2.22-2.25). Then $\mathcal{U}^{\tau}$ satisfies the following entropy inequality:

$$
\partial_{t} \eta\left(\mathcal{U}^{\tau}\right)+\partial_{x}\left(u^{\tau} \eta\left(\mathcal{U}^{\tau}\right)\right) \leq-\frac{\nu_{e i}}{k_{B} T_{i}^{\tau} T_{e}^{\tau}}\left(T_{i}^{\tau}-T_{e}^{\tau}\right)^{2}-\tau \frac{5 k_{B}}{2} \sum_{\alpha=e, i} \frac{1}{m_{\alpha}} \partial_{x}\left(n_{\alpha}^{\tau} \partial_{x} T_{\alpha}^{\tau}\right)
$$

where $\eta$ is defined by (1.4).

Proof. The result is obtained by multiplying (2.22)-(2.25) by $\eta^{\prime}\left(\mathcal{U}^{\tau}\right)$. The system $(2.22)-(2.25)$ being written in the synthetic form (1.3), we denote $W$ the viscous terms

$$
W=u^{\tau} \partial_{x}\left(J\left(\mathcal{U}^{\tau}\right) \partial_{x} \mathcal{U}^{\tau}\right)+\partial_{x}\left(D\left(\mathcal{U}^{\tau}\right) \partial_{x} \mathcal{U}^{\tau}\right)
$$

In [1] we have shown that

$$
\eta^{\prime}\left(\mathcal{U}^{\tau}\right)\left[\partial_{t} \mathcal{U}^{\tau}+A\left(\mathcal{U}^{\tau}\right) \partial_{x} \mathcal{U}^{\tau}-S\left(\mathcal{U}^{\tau}\right)\right]=\frac{\nu_{e i}}{k_{B} T_{i}^{\tau} T_{e}^{\tau}}\left(T_{i}^{\tau}-T_{e}^{\tau}\right)^{2} .
$$

It remains to prove that

$$
\eta^{\prime}\left(\mathcal{U}^{\tau}\right) W \leq-\frac{5 k_{B}}{2} \sum_{\alpha=e, i} \frac{1}{m_{\alpha}} \partial_{x}\left(n_{\alpha}^{\tau} \partial_{x} T_{\alpha}^{\tau}\right)
$$


Note that the first component of $W$ is equal to zero, so that $\partial_{\rho} \eta$ is not needed. We have

$$
\begin{aligned}
\eta^{\prime}(\mathcal{U})= & \left(\partial_{1} \bar{\eta}_{e}\left(\rho c_{e}, \varepsilon_{e}\right), \partial_{2} \bar{\eta}_{e}\left(\rho c_{e}, \varepsilon_{e}\right)\right)\left(\begin{array}{cccc}
c_{e} & 0 & 0 & 0 \\
-\frac{\mathcal{E}_{e}}{c_{e} \rho^{2}}+\frac{q^{2}}{\rho^{3}} & -\frac{q}{\rho^{2}} & \frac{1}{\rho c_{e}} & 0
\end{array}\right) \\
& +\left(\partial_{1} \bar{\eta}_{i}\left(\rho c_{i}, \varepsilon_{i}\right), \partial_{2} \bar{\eta}_{i}\left(\rho c_{i}, \varepsilon_{i}\right)\right)\left(\begin{array}{cccc}
c_{i} & 0 & 0 & 0 \\
-\frac{\mathcal{E}_{i}}{c_{i} \rho^{2}}+\frac{q^{2}}{\rho^{3}} & -\frac{q}{\rho^{2}} & 0 & \frac{1}{\rho c_{i}}
\end{array}\right) .
\end{aligned}
$$

Hence, denoting $q=\rho u$,

$$
\partial_{q} \eta(\mathcal{U})=\frac{u}{k_{B}}\left(\frac{c_{e}}{\varepsilon_{e} m_{e}\left(\gamma_{e}-1\right)}+\frac{c_{i}}{\varepsilon_{i} m_{i}\left(\gamma_{i}-1\right)}\right), \quad \partial_{\mathcal{E}_{\alpha}} \eta(\mathcal{U})=\frac{-1}{\varepsilon_{\alpha} m_{\alpha}\left(\gamma_{\alpha}-1\right)}, \alpha=e, i .
$$

As $p_{\alpha}=n_{\alpha} k_{B} T_{\alpha}$, this can be written as

$$
\partial_{q} \eta(\mathcal{U})=\frac{u}{k_{B}}\left(\frac{c_{e}}{T_{e}}+\frac{c_{i}}{T_{i}}\right), \quad \partial_{\mathcal{E}_{\alpha}} \eta(\mathcal{U})=\frac{-1}{k_{B} T_{\alpha}}, \quad \alpha=e, i .
$$

Now we multiply $\eta^{\prime}(\mathcal{U})$ by $W$, that is :

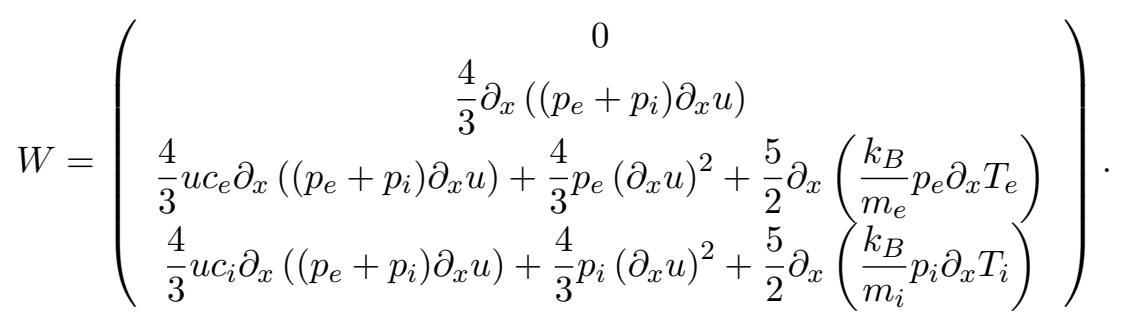

Therefore

$$
\begin{aligned}
\eta^{\prime}(\mathcal{U}) W & =-\frac{4}{3 k_{B}}\left(\partial_{x} u\right)^{2}\left(\frac{p_{e}}{T_{e}}+\frac{p_{i}}{T_{i}}\right)-\frac{5}{2 k_{B} T_{e}} \partial_{x}\left(\frac{k_{B}}{m_{e}} p_{e} \partial_{x} T_{e}\right)-\frac{5}{2 k_{B} T_{i}} \partial_{x}\left(\frac{k_{B}}{m_{i}} p_{i} \partial_{x} T_{i}\right) \\
& =-\frac{4 n}{3}\left(\partial_{x} u\right)^{2}-\sum_{\alpha=e, i}\left(\frac{5}{2 m_{\alpha} T_{\alpha}} \partial_{x}\left(n_{\alpha} k_{B} T_{\alpha} \partial_{x} T_{\alpha}\right)\right) .
\end{aligned}
$$

Using the fact that

$$
T_{\alpha}^{-1} \partial_{x}\left(n_{\alpha} T_{\alpha} \partial_{x} T_{\alpha}\right)=\partial_{x}\left(n_{\alpha} \partial_{x} T_{\alpha}\right)+\frac{n_{\alpha}\left(\partial_{x} T_{\alpha}\right)^{2}}{T_{\alpha}}
$$

we thus have

$$
\eta^{\prime}(\mathcal{U}) W=-\frac{4 n}{3}\left(\partial_{x} u\right)^{2}-\frac{5 k_{B}}{2} \sum_{\alpha=e, i} \frac{1}{m_{\alpha}}\left(\partial_{x}\left(n_{\alpha} \partial_{x} T_{\alpha}\right)+\frac{n_{\alpha}\left(\partial_{x} T_{\alpha}\right)^{2}}{T_{\alpha}}\right) .
$$




\section{Conclusion}

In this paper, starting from a kinetic model, we have derived a viscous approximation of the bitemperature Euler system from a Chapman-Enskog expansion. We have been able to compute explicitly all the viscous terms and we have obtained a generalization of the model proposed in [6]. Then we have proved an entropy inequality. These results support the approach taken in [1] where the same kinetic model is the basis of the numerical approximation of the system (1.2). The case $\gamma_{e} \neq \gamma_{i}$ can be handled by using a kinetic model with internal energy variable (see Remark 1). In a future work we plan to study the shocks obtained by limits of travelling waves constructed from this viscous model and to compare them to the ones numerically computed in our previous work [1].

\section{References}

[1] Aregba, D., Breil, J., Brull, S., Dubroca, B., and Estibals, E. Modelling and numerical approximation for the nonconservative bitemperature Euler model. Math Models and Numerical analysis 52, 4 (2018), 1353-1383.

[2] Aregba, D., And Brull, S. Construction and approximation of the polyatomic bitemperature Euler system. Preprint, 2017.

[3] Berthon, C., And Coquel, F. Nonlinear projection methods for multi-entropies NavierStokes systems. Math of Comp. 76 (2007), 1163-1194.

[4] Bouchut, F. Construction of BGK models with a family of kinetic entropies for a given system of conservation laws. J. Statist. Phys. 95, 1-2 (1999), 113-170.

[5] Brull, S., Dubroca, B., And LhÉBrard, X. Modelling and entropy satisfying relaxation for the nonconservatioce Euler system with transverse magnetic. Preprint (2018).

[6] Chalons, C., And Coquel, F. Navier-Stokes equations with several independent pressure laws and explicit predictor-corrector schemes. Numerisch Math. 103, 3 (2005), 451-478.

[7] Coquel, F., And Marmignon, C. Numerical methods for weakly ionized gas. Astrophysics and Space Science 260, 1-2 (1998), 15-27.

[8] Cuesta, C. M., Hittmeir, S., And Schmeiser, C. Kinetic shock profiles for nonlinear hyperbolic conservation laws. Riv. Mat. Univ. Parma (8) 1 (2009), 139-198.

[9] Dal Maso, G., Le Floch, P., and Murat, F. Definition and weak stability of nonconservative products. J. Math. Pures et Appl 74 (1995), 483-548.

[10] Graille, B., Magin, T., And Massot, M. Kinetic theory of plasmas:. Maths models and methods in the Appl. Sci. 19 (2009), 527-599.

[11] Raviart, P., And Sainsaulieu, L. A nonconservative hyperbolic system modeling spray dynamics. I. Solution of the Riemann problem. Math. Models Meth. in Appl. Sciences 5 (1995), 297-333. 
[12] Sainsaulieu, L. Ondes progressives solutions de systèmes convectifs-diffusifs et systèmes hyperboliques non conservatifs. C.R. Acad. Sci. Paris 312 (1991), 491-495.

[13] Sainsaulieu, L. Equilibrium velocity distribution functions for a kinetic model of twophase flows. Math. Models Meth. in Appl. Sciences 5 (1995), 191-211.

[14] Serre, D. Systems of conservation laws. 1. Cambridge University Press, Cambridge, 1999. Hyperbolicity, entropies, shock waves, Translated from the 1996 French original by I. N. Sneddon.

[15] Wargniez, Q., Faure, S., Graille, B., Magin, T., and Massot, M. Numerical treatment of the nonconservative product in a multiscale fluid model for plasmas in thermal nonequilibrium: application to solar physics. Preprint (2018). 